\title{
La formación de administradores en el nuevo entorno internacional
}

\author{
José Luis Pariente Fragoso*
}

\begin{abstract}
Resumen
La formación que reciben los estudiantes de administración en México no va acorde con las tendencias y las exigencias que demandan los nuevos entornos internacionales. En este artículo se hace un análisis de los programas de licenciatura en Administración que se imparten en veintidós universidades mexicanas, reconocidas por la agencia oficial certificadora en esa disciplina (CACECA) para comprobar la hipótesis de que los actuales currículos están estructurados con base en el proceso administrativo tradicional y sus áreas funcionales, mientras que las materias relacionadas con las habilidades que un profesional de la administración debería tener en este mundo globalizado (como el liderazgo, la toma de decisiones, el conocimiento de otras culturas y la creatividad, por citar algunas de las más señaladas por los expertos en la materia) sólo figuran de manera marginal en los programas de estudio de las carreras certificadas en el nivel nacional.

Palabras clave: formación de administradores, nuevos entornos administrativos, currículo en administración, programas de estudio en administración, licenciatura en Administración.
\end{abstract}

\section{Introducción}

En un reciente artículo de Warren G. Bennis y James O'Toole (2005), publicaCdo en la edición en español de la Harvard Business Review, los autores sostienen la tesis de que las escuelas de negocios, preocupadas de manera creciente por la investigación científica, van por el camino equivocado al contratar profesores con una limitada experiencia en el mundo real y, como resultado, forman alumnos

\footnotetext{
* Director General de Innovación Tecnológica de la Universidad Autónoma de Tamaulipas. Correo electrónico: jpariente@uat.edu.mx
} 
que se gradúan mal preparados para lidiar con los verdaderos asuntos que atañen a la gestión; es decir, los asuntos complejos y no cuantificables. Asimismo, afirman que destacados profesores tienen otra percepción del asunto, como Henry Mintzberg, de la McGill University, quien señala que "[...] el principal culpable son los planes de estudios poco relevantes (p. 86)"; aunado a ello, Bennis y O'Toole sostienen:

La verdadera causa de la actual crisis en la educación de negocios tiene un alcance mucho más amplio y se puede remontar al dramático giro que experimentó la cultura de las escuelas de negocios. A lo largo de las últimas décadas, muchas escuelas de negocios lideres han adoptado de manera silenciosa un modelo inapropiado de excelencia académica, que en última instancia es contraproducente. En vez de medirse a sí mismas por la competencia de sus graduados, o por qué tan bien entienden sus profesores los impulsores del desempeño empresarial, se miden casi exclusivamente por el rigor de sus investigaciones cientificas. (p. 86).

Aunque coincidimos con los autores en su apreciación general del problema, en el caso de nuestro país, en donde esta presión por la investigación apenas empieza a sentirse - derivada de los procesos de certificación y el reconocimiento del profesorado en cuerpos académicos e instituciones como el PROMEP o el Sistema Nacional de Investigadores- pensamos, más en concordancia con el profesor Mintzberg, que en México actualmente el verdadero problema radica en la estructura de los planes de estudio que, por más de cincuenta años, han girado en torno a un modelo totalmente obsoleto que descansa en dos ejes fundamentales alrededor de los cuales se articula todo el desarrollo del currículo: el proceso administrativo y las áreas funcionales de la administración.

Partimos del convencimiento de que la administración es, antes que nada, una profesión; considerarla una disciplina académica o, peor aún, una ciencia — pareciera que la máxima aspiración de todas las profesiones hoy en día es ser consideradas como ciencias - ha conducido a prácticas erróneas en los diseños curriculares $\mathrm{y}$ en el mismo ejercicio profesional, como bien afirman Bennis y O'Toole:

Lo que nosotros denominamos el modelo cientifico se basa en el erróneo supuesto que los negocios es (sic) una disciplina académica, tal como lo es la química o la geología. Pero los negocios son, en realidad, una profesión, semejante a la medicina o las leyes, y las escuelas de negocios son escuelas profesionales, o deberían serlo. Al igual que otras profesiones, los negocios reúnen el trabajo de muchas disciplinas 
La formación de administradores

en el nuevo entorno internacional

académicas (. . .). A nuestro juicio, ninguna reforma al plan de estudios funcionará a menos que el modelo científico sea reemplazado por un modelo más apropiado y que se base en los requerimientos especiales de la profesión. (p. 86).

Derivado de lo anterior, la hipótesis central del presente trabajo es que los actuales planes de estudio de las licenciaturas en Administración en México están articulados en función de los procesos administrativos tradicionales y el estudio de cuatro o cinco de las áreas denominadas "funcionales" de la administración. Esta estructura curricular se complementa con una serie de materias básicas de carácter cuasipropedéutico y otro conjunto de materias especializadas, ya sean optativas o pertenecientes a un núcleo introductorio, que dependen más del capricho de las autoridades universitarias en turno o de las modas administrativas, que de un análisis sustentado en las necesidades de los mercados de trabajo o las realidades locales, regionales o nacionales.

\section{Antecedentes}

Diversos estudiosos y académicos nacionales han abordado el problema de la formación de los administradores desde diversos puntos de vista y enfoques, posiciones que contemplan tanto la necesidad de adecuar los planes y programas a la idiosincrasia del pueblo mexicano (Ríos, 1989), como a los problemas derivados de la ausencia de investigaciones y publicaciones en el campo (Montaño, 1999), la formación en valores (López Garza y Zubillaga, 1998), la influencia de las tendencias políticas y sociales (Solís, 1999; Vargas, s/f) e incluso, el impacto de las nuevas tecnologías de la informática y las telecomunicaciones en los programas de estudio en las instituciones de educación superior en el país.

De igual forma, preocupados por la situación imperante, también han propuesto recomendaciones específicas para tratar de elevar la calidad de las licenciaturas, entre las que se mencionan, de manera consistente, la de "contar con planes de estudio flexibles, los cuales estén en un proceso permanente de actualización " (De Agüero, 2003, p. 42), inclusive se sugieren perfiles que vayan más allá de un currículo basado en las funciones y los procesos administrativos tradicionales. Al respecto, afirma Raymundo Soto (1999, párrafo 33):

Actualmente, las organizaciones empiezan a demandar un nuevo perfil de administrador, a quienes se les requiere que sean altamente competitivos no sólo desde el punto de vista de las competencias técnicas del procesos administrativo y 
las áreas funcionales de la empresa, sino que también posean y desarrollen visión holística de los problemas humanos, sociales, económicos y organizacionales que enfrenta el país, así como conciencia del cambio y pensamiento sistémico.

Con respecto a la necesidad, cada vez más imperiosa, de incorporar a los planes y programas de estudio un conocimiento más sólido acerca de la realidad nacional y del carácter y comportamiento del mexicano, ya desde hace casi dos décadas Jorge Ríos Szalay apuntaba textualmente: "Los planes de estudio de la licenciatura en Administración nunca han incluido el análisis de las formas de organización social hoy vigentes en el 'México profundo' para millones de mexicanos, como son aquellas existentes para organizar el trabajo, el gobierno comunal y el intercambio." (Ríos, 1989, p. 57). Este mismo autor proponía en su texto (pp. 84-99) algunos elementos culturales propios del mexicano que debían aprovecharse para una mejor formación administrativa:

a) Concepción de la naturaleza y ubicación que se da al hombre en el cosmos.

b) Concepción del recurso tierra

c) Tendencia a la autosuficiencia

d) Capacitación individual multifacética

e) Sentido de la solidaridad social

f) Participación democrática

Sin embargo, los nuevos entornos internacionales han añadido nuevos elementos que, de manera imperiosa, deberán incluirse en los planes de estudio. De acuerdo con Pedro Solís (1999) existen en la actualidad importantes retos para el administrador, derivados de las siguientes tensiones emergentes:

a) Tensión entre lo global y lo local.

b) Tensión entre la regulación por la competencia del mercado o por la cooperación.

c) Tensión entre las "ciencias duras" y las "ciencias blandas".

El conocimiento y cuidado del entorno, con el objetivo prioritario de poder legar a las futuras generaciones un mundo mejor que el que nos fue entregado, sigue siendo preocupación fundamental en la formación de los nuevos administradores, incluso ha motivado la incorporación de materias específicas en los planes de 
La formación de administradores

en el nuevo entorno internacional

estudio de algunas universidades, por ejemplo, las materias de Medio Ambiente y Desarrollo Sustentable (Gutiérrez y Medina, 2003) o Tamaulipas, los retos del desarrollo (Navarro y Pariente, 2001), que son obligatorias para los estudiantes, además de los de administración, de todas las licenciaturas que se imparten en la Universidad Autónoma de Tamaulipas.

Otro serio problema que aqueja a los programas de administración es la presencia de bibliografía de origen norteamericano en su mayor parte, en donde se presentan modelos administrativos que responden a situaciones en ocasiones totalmente distintas a nuestra realidad mexicana. La posible causa de escasez de bibliografía se debe a la reducida cantidad de investigaciones hechas por autores mexicanos. Este problema se agrava aún más cuando nuestros estudiantes, debido a los tradicionales procesos de enseñanza-aprendizaje que aún imperan en nuestras aulas, no desarrollan la necesaria capacidad de recontextualización de modelos desarrollados en otras latitudes.

La ausencia de textos escritos por autores nacionales, que no sean sólo traducciones de obras en otros idiomas, principalmente en inglés, sino producciones derivadas de la propia experiencia local, ha sido preocupación constante de estudiosos mexicanos. Desde hace ya casi dos décadas, Jorge Ríos Szalay (1989, p. 58) apuntaba en uno de sus textos:

[...] la realidad predominante en las escuelas y facultades de administración del país es la de abocarse prioritariamente a las necesidades de las grandes empresas, y la de seguir acrítica y casi exclusivamente las corrientes administrativas en boga en los Estados Unidos. Una muestra de ello es la abrumadora presencia de obras extranjeras, principalmente del vecino país, en la bibliografia indicada en los planes de estudio.

Esta situación se agrava de manera particular cuando los modelos o procesos administrativos se tratan de implantar en entornos ajenos a los que propiciaron su desarrollo, como dejamos asentado en nuestro libro Teoría de las organizaciones. Un enfoque de metáforas:

Uno de los problemas que tiene que ver con el estudio de las organizaciones en nuestro medio, es que la mayoría de nuestros textos son traducciones, desgraciadamente poco afortunadas a veces, de libros editados en inglés. Aparte de los problemas de traducción, cuando se tocan los temas relacionados con el entorno de las organizaciones, se hacen referencias a ambientes que no son 
propiamente los nuestros. No es ni nuestra situación fisica, ni nuestra situación demográfica, ni la política, la social, la económica o la cultural; por lo tanto, la información que podemos obtener de ellos es relativa si queremos aplicarla a nuestro entorno particular en México (Pariente, 2001, p. 118).

En el caso de la investigación, las publicaciones y artículos de Luis Montaño presentan un vívido panorama de la precaria situación en que se encuentran los estudios administrativos y organizacionales; al respecto, afirma el autor en las conclusiones de uno de sus artículos (Montaño, 1999):

La Administración es una disciplina relativamente reciente en México, con poca tradición en investigación y orientada principalmente hacia las áreas docentes. Se enseñan generalmente modelos idealizados —desde la optimización hasta la excelencia — ; los cuales representan la parte "dura", "cientifica" relativamente fácil de transferir, pero difícil de operar. La Administración está asentada en organizaciones concretas, alejadas de las condiciones de idealidad de dichos modelos. No se puede estudiar la Administración en abstracto, ésta tiene que ser referida siempre a las condiciones particulares de las organizaciones que aplican esos dispositivos.

Afortunadamente, esta ausencia de investigaciones en el ámbito nacional empieza a revertirse gracias a los esfuerzos de los académicos mexicanos y las organizaciones profesionales que de manera creciente se han empeñado en propiciar espacios para la investigación, la presentación, la discusión y el análisis de las investigaciones nacionales. Dos mecanismos han contribuido de manera notable a este propósito: por un lado, los programas doctorales en estudios organizacionales y en administración, que con la exigencia de sus disertaciones están contribuyendo a la generación y publicación de importantes documentos para el conocimiento de la realidad organizacional y administrativa nacional; por otro, los congresos nacionales e internacionales en estas disciplinas entre los que destacan, de manera importante, los organizados por la Academia de Ciencias Administrativas (ACACIA), que tendrá su décima edición en 2006 en la Universidad Autónoma de San Luis Potosí; el Congreso Internacional de Contaduría, Administración e Informática con su próximo XI Foro de Investigación, organizado por la Facultad de Contaduría y Administración de la UNAM y la ANFECA, y el Congreso Internacional de Análisis Organizacional, a cargo del posgrado de estudios Organizacionales de la Universidad Autónoma Metropolitana-Iztapalapa en coordinación con otras instituciones educativas nacionales y extranjeras. 
La formación de administradores

en el nuevo entorno internacional

Una de las principales críticas que han recibido los actuales programas de formación de administradores es la falta de pertinencia con las demandas de las organizaciones productivas y de servicio, que exigen de sus futuros empleados habilidades y conocimientos que en ocasiones resultan muy alejados de lo que aprenden en las aulas. Por otro lado, lo que realmente hacen los ejecutivos en su vida cotidiana ya fue motivo de un demoledor estudio para la tesis doctoral de un brillante estudiante canadiense que demostró, en un trabajo posteriormente publicado como libro con el título The Nature of Managerial Work (La naturaleza del trabajo administrativo), que lo que realmente hacen estos profesionales se aleja mucho del proceso administrativo tradicional. Henry Mintzberg probó que las habilidades y conocimiento de los ejecutivos tenían más que ver con las habilidades interpersonales, la toma de decisiones y la solución de problemas, las redes, las relaciones externas e incluso con el hecho de ser sólo una figura decorativa con los procesos tradicionales de planeación, organización, dirección y control.

Algunas de las propuestas que se han hecho para la reestructuración de los planes y programas de estudio en administración parten de criterios utilitaristas, como los derivados de las certificaciones ISO o los premios de calidad como el Baldrige (2005) que para el caso de los negocios plantea los siguientes criterios de valuación:

1. Liderazgo

2. Planeación estratégica

3. Enfoque en el cliente y el mercado

4. Medición, análisis y administración del conocimiento

5. Enfoque en los recursos humanos (sic)

6. Administración de procesos

7. Resultado de negocios

No obstante lo anterior, es de importancia capital no perder de vista que si bien, como ya hemos apuntado anteriormente, la administración es básicamente una profesión; el profesional es, antes que nada, un ser humano que busca su propio desarrollo en armonía con los demás y con su entorno.

Por otra parte, tenemos que la palabra profesión proviene del latín professio, onis, una de las acepciones que presenta el diccionario de la Real Academia Española se centra en el empleo, facultad u oficio que alguien ejerce y por el que percibe una retribución; podemos, por tanto, considerar a un profesional como 
aquella persona que ejerce una profesión con suficiente capacidad y habilidades al servicio del bien común dentro de una norma de conducta ética aceptada y que percibe, por ello, una remuneración y un estatus social determinado.

De acuerdo con Greenwood (citado en Hinings, 2001) las características de una profesión son las siguientes:

1. Teoría sistemática como base de conocimiento

2. Formación prolongada y certificación

3. Autoridad reconocida

4. Sanción de la comunidad y legitimación

5. Cultura profesional, que comprende un conjunto de valores que incluyen el juicio de los pares, la vocación, el servicio público, la autoregulación y la autonomía.

6. Códigos éticos

Si bien el concepto de profesión comenzó a utilizarse en Inglaterra desde el siglo XVI para designar la actividad laboral relativa a tres campos específicos: teología, derecho y medicina, su uso se ha extendido rápidamente a otras ramas del saber humano; en la actualidad, y con base en Fernández Pérez (2001, p. 35) se afirma:

El contexto de la globalización y la regionalización de los servicios profesionales están condicionando un nuevo modelo de profesiones para el siglo XXI, las cuales tendrán que asumir elementos que respondan a una nueva sociedad que día a día cambia. Estas transformaciones están propiciando que las profesiones incorporen, para su desarrollo, algunos aspectos como la acreditación de planes y programas de estudio de educación superior, la certificación y la actualización continua de profesionistas, la vinculación de los colegios y asociaciones profesionales con las instituciones de educación superior y flexibilidad en el trabajo, entre otros.

Componente importante en los procesos de formación de los profesionales son los asuntos éticos reconocidos por las propias agrupaciones profesiones en sus códigos de comportamiento; si bien estamos convencidos de que tanto en la ética como en otras disciplinas necesarias para el administrador el "currículo oculto" juega un papel predominante, la escolarización de la enseñanza en valores, así como de la responsabilidad social de las empresas y las organizaciones en general, es un asunto que ha estado asumiendo un papel importante en los años recientes (Blanchard y O'Connor, 1997; Llano, 1997; Frederick, 2001; García y Dolan, 1997). 
La formación de administradores

en el nuevo entorno internacional

En 1997, Ken Blanchard y Michael O'Connor acuñaron el término Managing by Values, que también por esos años utilizaron García y Dolan como título para su libro Dirección por valores. La dirección por valores $(\mathrm{DpV})$ es una herramienta de liderazgo que va más allá de los conceptos de dirección por instrucciones y dirección por objetivos que la precedieron, y pretende darle un sentido más ético y emocional a la construcción de empresas.

Por último, la irrupción avasalladora de las nuevas tecnologías de la información y las telecomunicaciones (TICs) ha propiciado la generación de nuevos conceptos y corrientes administrativas y organizacionales — basadas en la administración de la información y el trabajo en redes - en entornos ajenos a los espacios tradicionales en los que las organizaciones llevaban a cabo sus tareas rutinarias. Nuevos conceptos como el teletrabajo, el comercio electrónico, la gestión del conocimiento y la administración del cambio o de la complejidad, se incluyen cada vez con mayor frecuencia en los programas de estudio de los posgrados en administración y estudios organizacionales.

\section{Método}

Con objeto de comprobar la validez de nuestra hipótesis de trabajo, recabamos la información de los planes de estudio correspondiente a las veintidós instituciones de educación superior certificadas — hasta el momento de escribir este artículopor el Consejo de Acreditación en la Enseñanza de la Contaduría y Administración, A.C. (CACECA) de acuerdo con los datos que figuran en la tabla 1 (anexo 1). Cabe hacer la aclaración de que estamos conscientes que muchos de los esfuerzos por mejorar los planes de estudio se hacen en ocasiones de manera marginal en instituciones aisladas que tienen visiones acordes con el papel del administrador en los nuevos entornos; pero el propósito del presente artículo es demostrar, precisamente, que son la mayoría de las universidades, y en especial las más acreditadas, las que menos incorporan estas tendencias innovadoras. Los resultados corresponden a instituciones de educación superior (IES) de quince estados de la república que abarcan tanto organizaciones públicas como privadas e incluyen desde la Universidad Nacional Autónoma de México y el Instituto Politécnico Nacional, hasta el Instituto Tecnológico y de Estudios Superiores de Monterrey y que pudieran ser consideradas, por el hecho de tener certificados sus programas de estudio, como representativas de lo más avanzado en materia de administración en el país. 
Una vez identificadas las instituciones se localizaron los planes de estudio de las licenciaturas correspondientes, para ello se utilizó la internet como medio de búsqueda. Afortunadamente, todas las instituciones investigadas tienen la información disponible en línea, por lo que el siguiente paso fue concentrar los datos correspondientes a todas las materias de los planes de estudio en administración - o licenciaturas equivalentes - en una matriz que, posteriormente y para fines de análisis, se dividió en tres tablas separadas. Cabe señalar que las tablas correspondientes se encuentran al final de este artículo en los siguientes anexos: Materias básicas (anexo 2), Proceso administrativo y áreas funcionales (anexo 3) y Otras materias (anexo 4).

Con los datos ya tabulados, las materias se ordenaron por frecuencia de aparición y se procedió al análisis de las tablas; los resultados se presentan en el siguiente apartado; por último, se redactaron las conclusiones y algunas recomendaciones preliminares derivadas de las mismas y la contrastación con los modelos y conceptos teóricos estudiados.

Es importante aclarar que las materias se agruparon por los nombres con los que aparecen en los programas de estudio sin conocer sus contenidos específicos. Lo anterior puede ser una limitante en el estudio porque en ocasiones el nombre de la materia no refleja con fidelidad los contenidos específicos de la misma.

\section{Análisis de los resultados}

Para este análisis, los datos obtenidos de dividieron en tres grandes bloques (representados en los anexos 2, 3 y 4). En el primero de ellos se agrupan las materias que hemos denominado básicas; es decir, aquellas que resulten introductorias o de índole disciplinar que pretenden que el alumno adquiera los conocimientos generales de una disciplina específica como puede ser la contabilidad, las matemáticas o el derecho; se incluyen también las relacionadas con la computación y la informática. En el segundo bloque agrupamos las materias relacionadas tanto con el proceso administrativo tradicional: planeación, organización, dirección y control, como las que se refieren a las áreas funcionales; se incluyen en este apartado mercadotecnia, producción e investigación de operaciones, finanzas, recursos humanos, compras, ventas y relaciones públicas. En el último apartado figuran todas aquellas materias que no se incluyen en los apartados anteriores y que abar- 
La formación de administradores

en el nuevo entorno internacional

can una amplia gama de disciplinas relacionadas con los aspectos metodológicos, de habilidades del pensamiento y de comunicación, de conocimiento del entorno o de algunas tendencias administrativas actuales, como puede ser el caso de las PYMES o la administración de la calidad.

\section{Materias básicas}

En el anexo 2 puede observarse como prácticamente todos los planes de estudio incluyen las siguientes materias: Introducción a la Administración, Matemáticas y Estadística, Economía, Contabilidad, Derecho, Computación e Informática y Sociología o Sociología de la Organización; este último caso sólo se incluye en 12 de los planes de estudio analizados.

\section{Proceso administrativo}

El anexo 3 incluye los dos bloques de materias que constituyen los ejes fundamentales alrededor de los cuales se estructuran prácticamente todos los programas de estudios analizados. En el primero de ellos se agruparon las materias correspondientes al proceso administrativo tradicional, a saber: planeación, organización, dirección y control, a las que también hemos añadido las relacionadas con la auditoría (entendida ésta como un proceso de control) y las materias que analizan el proceso administrativo como un todo. En la tabla de este anexo 3 se puede observar que la mayoría de los programas incluyen la materia de planeación, así como la de control y/o auditoría, aunque en el caso de dirección el porcentaje comienza a decrecer hasta llegar a sólo nueve programas que incluyen la materia de organización. En el caso del proceso administrativo como un todo sólo ocho de los programas analizados incluyen esa materia, es decir, un $36 \%$ del total.

Por otra parte, las áreas funcionales tradicionales están ampliamente incluidas en todos los programas: Mercadotecnia figura en todos los programas, Producción y/ o Investigación de Operaciones, Finanzas y Recursos Humanos no aparecen en dos de los programas. El porcentaje desciende hasta un $54 \%$ en el caso de Compras y a un 31\% para la materia de Ventas, Relaciones Humanas sólo se imparte con ese nombre en tres de los programas analizados (13\%). 
José Luis Pariente Fragoso

\section{Otras materias}

En el anexo 4 tenemos una tabla que agrupa 21 materias que se encuentran mínimo en tres ocasiones en los diferentes planes de estudio; asimismo, existen otras materias con una frecuencia menor como es el caso de Administración Internacional, que sólo aparece con ese nombre en dos de los programas, o Cultura Organizacional, que sólo figura en uno de ellos.

Las materias que registran una frecuencia mayor al 50\% son Metodología de la Investigación, Desarrollo de Negocios y/o Emprendedores, Ética y Comportamiento Organizacional; mientras que con menor incidencia tenemos Comercio Exterior, Administración de la Calidad, PYMES, Inglés y Administración Pública (estas cuatro últimas con un 36\%); en menor grado aparecen materias como Psicología, Comunicación Organizacional, Administración del Entorno, Habilidades del Pensamiento, Comunicación Oral y Escrita, Diseño Organizacional, Impuestos y Desarrollo Organizacional; las materias que figuran con menos frecuencia (20\%) fueron, paradójicamente, Toma de Decisiones, Comercio Electrónico y Liderazgo; es decir, algunas de las que se relacionan más con las habilidades que los entornos internacionales reclaman para la formación de los modernos administradores.

De manera complementaria, del análisis detallado de los programas de estudio, surgen algunas observaciones generales que resumimos a continuación.

En primer lugar, destaca la inclusión creciente de algunos cursos aislados o incluso módulos complementarios de materias denominadas de distinta forma (materias sello, bloque de identidad universitaria, área de desarrollo integral o materias co-curriculares) con las cuales se pretende que los alumnos adquieran algunos conocimientos básicos relacionados con los idiomas, las habilidades del pensamiento, los valores, o bien, con el conocimiento de su propia realidad regional y estatal como es el caso de las materias Introducción al Pensamiento Universitario (UPAP), Universidad y Conocimiento (UACH), o Tamaulipas y los Retos del Desarrollo (UAT). Otras instituciones ya utilizan nuevos términos en sustitución del obsoleto y poco apropiado concepto de recursos humanos e incorporan materias denominadas Capital Intelectual (IPN), Capital Humano (ITC) o Factor Humano (UVM). También se empieza a notar la preocupación por el estudio de los organismos internacionales (UDG) e incluso los Estudios Organizacionales 
La formación de administradores

en el nuevo entorno internacional

(UAEM) o el Aprendizaje Organizacional (UAEH). Sin embargo, todos estos intentos surgen todavía de manera aislada y no constituyen una parte importante en el núcleo de los programas de las licenciaturas; también es importante destacar que prácticamente en todos los planes de estudio tenemos la ausencia de cursos relacionados con el aprendizaje y la aplicación de los métodos cualitativos.

En la mayoría de los casos las materias más relacionadas con los nuevos modelos de gestión o las herramientas administrativas más actuales se ofrecen como materias optativas y no integrando el núcleo central de los programas, tal es el caso de la Administración de la Calidad (UACJ), Innovaciones Técnicas de la Administración o Emprendedores (UNAM) o el de Liderazgo dentro de los cursos sello del ITESM.

Por estar en concordancia con un criterio que ya habíamos sostenido en un artículo anterior (Pariente, 2001) como importante estrategia por desarrollar en los administradores en entornos multiculturales, mención especial merece el caso de la UANL, que ha incorporado la materia de Apreciación de las Artes como parte de su programa de formación integral.

Por último, es notoria la ausencia —en la mayoría de los programas- de algunas materias relacionadas con el uso de las nuevas tecnologías de la informática y las telecomunicaciones, que actualmente están cobrando importancia en la gestión de las organizaciones contemporáneas como son el teletrabajo y el trabajo en redes, el comercio electrónico, la gestión del conocimiento y la administración del cambio y de la complejidad.

\section{Conclusiones y recomendaciones}

Como conclusión del análisis de los datos obtenidos se comprueba la hipótesis planteada de que la estructura de los programas de estudio de las licenciaturas en Administración en México, representadas por aquéllas certificadas por CACECA, tienen como eje central de su diseño curricular las funciones y el proceso administrativo tradicional. Prácticamente todas ellas incluyen un núcleo de materias básicas en las áreas disciplinares de las matemáticas, la informática, la contabilidad, la economía y el derecho, así como una serie de materias complementarias de la más diversa índole a veces agrupadas en un núcleo inicial formativo o como opciones optativas de la más variopinta conformación. 
En clara contraposición con las sugerencias de los especialistas en la disciplina y con los discursos oficiales y los derivados de las exigencias del mercado de trabajo, así como de los entornos internacionales, las materias de vanguardia que deberían integrar la currícula de los estudios administrativos —como liderazgo, solución de problemas y toma de decisiones, creatividad, trabajo en redes, conocimiento del entorno e incluso calidad total - sólo se encuentran de manera aislada y marginal en la mayor parte de todos los programas estudiados.

Con objeto de ser propositivos y a guisa de plantear sólo algunos apuntes preliminares para una solución a esta preocupante problemática para el desarrollo de la administración en el país (el tema de la especificidad de la formación de administradores en el nuevo entorno internacional será objeto de un trabajo posterior) estamos convencidos de que una solución que abogue por una reestructuración a los planes y programas de estudio existentes no solucionaría el problema de fondo si se mantiene el actual paradigma de diseño curricular. Debemos enseñar a nuestros estudiantes, siguiendo el pensamiento de Schein (1982), "[...] a convertirse en verdaderos agentes de cambio, sean cuales fueran sus especialidades. Debemos enseñarles cómo influir sobre sus organizaciones, desde cargos de escaso poder, sin sacrificar en el proceso sus valores profesionales."

Sin embargo, para lograr lo anterior y para poder consolidar un verdadero y sustantivo cambio en la formación de nuestros futuros administradores es imprescindible partir de un nuevo paradigma que deseche radicalmente los obsoletos enfoques de funciones y proceso administrativo además deberá articularse con una visión realista de los entornos multiculturales y globalizadotes de la sociedad en la que se desenvolverán los futuros profesionales de la administración en México y en el mundo. También este nuevo paradigma deberá ir acompañado, de manera indisoluble, por un importante viraje en los tradicionales procesos de enseñanza-aprendizaje en estas disciplinas y que haga énfasis en conceptos tales como:

1. Pertinencia con las necesidades y los requerimientos específicos de los mercados de trabajo y las demandas de la sociedad en su conjunto.

2. Formación con énfasis en la necesidad de una educación permanente.

3. Estudio de las organizaciones como entes sociales flexibles y paradójicos, en los cuales se llevan a cabo los procesos administrativos para el logro de objetivos comunes. 
La formación de administradores

en el nuevo entorno internacional

4. Inclusión, en todos los programas, de los métodos cualitativos, utilizados de manera creciente en las áreas administrativas y los estudios organizacionales.

5. Uso intensivo de las nuevas tecnologías de la información y las telecomunicaciones, así como el empleo alterno de programas de educación abierta y a distancia, como estrategia importante para poder competir con los programas de formación tanto de las empresas como de las cada vez más crecientes en número universidades empresariales.

6. Conocimiento del México actual y su potencial de desarrollo, así como de las regiones propias de los educandos, además de los elementos culturales que le son propios.

7. Conocimiento, no sólo de las lenguas extranjeras (como el inglés), sino de los modos de vida y la cultura de otros pueblos, particularmente a través del estudio de sus manifestaciones artísticas con una visión de tolerancia y respeto hacia lo diverso.

8. Estudio de las condiciones y los procesos naturales y conciencia de la necesidad de preservación de los mismos de una manera sostenible.

9. Énfasis en los valores universales, en especial la libertad, la democracia, la tolerancia y el respeto a los demás.

10.Redefinición del sentido del trabajo con la utilización de redes de cooperación y los mecanismos de negociación, colaboración y sentido comunitario, desde el ámbito local y regional hasta los entornos globales.

La administración es una profesión que no ha sabido adecuarse académicamente al ritmo acelerado del cambio organizacional en entornos cada vez más competitivos y globalizados. Los egresados de nuestras instituciones de educación superior en esta disciplina, para tener éxito como profesionales y lograr un pleno desarrollo personal, deberán contar con los conocimientos, las habilidades y los valores que reclaman los nuevos entornos internacionales, así como el uso de las nuevas tecnologías de la información y las telecomunicaciones, pero sin perder nunca de vista el origen y las tradiciones de su propia cultura nacional y local. Asimismo, estos egresados deberán poseer creatividad y capacidad para desenvolverse en un ambiente laboral en organizaciones cada vez más complejas, flexibles y multiculturales, preocupadas por la conservación de su entorno, con responsabilidad social y orientadas a la satisfacción de las necesidades de sus clientes y al desarrollo integral de su propios integrantes. 
José Luis Pariente Fragoso

\section{Referencias bibliográficas}

ACADEMIA DE CIENCIAS ADMINISTRATIVAS (ACACIA), consultado en noviembre de 2005: http://www.acacia.com.mx/; http:// academia.uat.edu.mx/acacia

BALDRIDGE NACIONAL QUALITY PROGRAM (2005), Criteria for Performance Excellence. Consultado en noviembre de 2005: http:// www.quality.nist.gov/Business_Criteria.htm

BENNIS, Warren G. y O’Toole, James (2005), ¿Cómo las escuelas de negocios perdieron el rumbo?", Harvard Business Review, 82(5), pp. 84-93.

BLANCHARD, K., O'Connors, M. \& Ballard, J. (1997), Managing by Values, San Francisco, CA: Berrett-Koehler Publishers

CONSEJO DE ACREDITACIÓN DE LA ENSEÑANZA EN CONTADURÍA Y ADMINISTRACIÓN, A.C. (CACECA), consultado en: http:// www.caceca.org/

DE AGÜERO AGUIRRE, Mario (2003), "La formación de administradores en el contexto actual", en Contaduría y Administración, No. 219, México: Facultad de Contaduría y Administración, UNAM.

FERNÁNDEZ PÉREZ, Jorge (2001), "Elementos que consolidan al concepto de profesión. Notas para su reflexión", en Revista Electrónica de Investigación Educativa, 3, (1) Ensenada, Baja California: Universidad Autónoma de Baja California, Instituto de Investigación y Desarrollo Educativo, consultado en: http://www.redie.ens.uabc.mx/vo13no2/contenidofernandez.html

FREDERICK, Robert E. (ed.). (2001), La ética en los negocios, México: Oxford.

GARCÍA, Salvador y Simón Dolan (1997), La dirección por valores, Madrid: McGraw Hill. 
La formación de administradores

en el nuevo entorno internacional

GUTIÉRREZ LOZANO, Joel y Teodoro Medina (coords.) (2003), Medio ambiente y desarrollo sustentable. Ciudad Victoria, México: Universidad Autónoma de Tamaulipas, Colección Misión XXI.

HININGS, C. R. (2001), "Professions in organizations", en Smelser, Neil J. \& Baltes, Paul B. (eds.), Internacional Enciclopedia of the Social \& Behavioral Sciences, Oxford: Elsevier.

LÓPEZ GARZA, Patricia y Ana Cristina Zubillaga (1998), "Desde una perspectiva humanista, ¿en qué valores formar al administrador que cruza el siglo XXI?" Revista Gestión y Estrategia, 14. México: Universidad Autónoma Metropolitana-Azcapotzalco, consultado en: http://www.azc.uam.mx/publicaciones/gestion/num14/doc16.htm

LLANO CIFUENTES, Carlos (1997), Dilemas éticos de la empresa contemporánea, México: Fondo de Cultura Económica.

MINTZBERG, Henry (1973), The Nature of Managerial Work, New York: Harper \& Row.

MONTAÑO HIROSE, Luis (1999, julio), "La investigación en administración. Reflexiones para el caso de México", en Administración y Organizaciones, 2, México: Universidad Autónoma Metropolitana-Xochimilco.

NAVARRO, Marco Aurelio y José Luis Pariente (coords.) (2001), Tamaulipas. Los retos del Desarrollo, Ciudad Victoria, México: Universidad Autónoma de Tamaulipas, Colección Misión XXI.

PARIENTE FRAGOSO, José Luis (2001), Teoría de las Organizaciones. Un enfoque de metáforas, México: Miguel Ángel Porrúa / CONACYT.

— (2001, julio), "Administración intercultural", en Administración y organizaciones, 3(6), México: Universidad Autónoma Metropolitana, pp. 95-108.

RÍOS SZALAY, Jorge (1989), La realidad cultural nacional y el estudio de la administración en México, México: Facultad de Contaduría y Administración, UNAM. 
SCHEIN, Édgar H. (1982), "Socialización organizacional y la profesión de la administración de empresas", en David A. Kolb (1982), Psicología de las organizaciones (Problemas contemporáneos). Madrid: Prentice Hall Internacional, consultado en: http://www.geocities.com/Athens/Crete/3108/ Socializacion.html

SOLÍS PÉREZ, Pedro C. (1999), "Los retos de las Ciencias Administrativas y de la Organización en el Siglo XXI", en Administración y Organizaciones, 2. México: Universidad Autónoma Metropolitana-Xochimilco.

SOTO SÁNCHEZ, Raymundo (1999), "La formación de administradores en la sociedad del conocimiento: retos y oportunidades", en Revista Gestión y Estrategia, 15. México: Universidad Autónoma MetropolitanaAzcapotzalco, consultado en: http://www.azc.uam.mx/publicaciones/ gestion/num15/doc06.htm

VARGAS HERNÁNDEZ, José Guadalupe (s/f), "Impacto de las tendencias políticas y sociales en la formación del capital humano con nivel de posgrado en ciencias organizacionales y administrativas", consultado en: http://www.gestiopolis.com/canales/economia/articulos/47/impacto.htm (q) 
La formación de administradores

en el nuevo entorno internacional

\section{Anexo 1}

\section{Programas académicos acreditados por CACECA}

\begin{tabular}{|c|c|c|c|c|}
\hline Estado & No. & Siglas & Institución & Licenciatura \\
\hline Aguascalientes & 1 & UAA & $\begin{array}{l}\text { Universidad Autónoma de } \\
\text { Aguascalientes }\end{array}$ & $\begin{array}{l}\text { Licenciatura en Administración } \\
\text { de Empresas }\end{array}$ \\
\hline \multirow[t]{2}{*}{$\begin{array}{c}\text { Baja } \\
\text { California }\end{array}$} & 2 & UABC & $\begin{array}{l}\text { Universidad Autónoma de Baja } \\
\text { California. Campus Ensenada, } \\
\text { Mexicali y Tijuana }\end{array}$ & $\begin{array}{c}\text { Licenciatura en Administración } \\
\text { de Empresas }\end{array}$ \\
\hline & 3 & CETYS & $\begin{array}{c}\text { Centro de Enseñanza Técnica y } \\
\text { Superior }\end{array}$ & $\begin{array}{c}\text { Licenciatura en Administración } \\
\text { de Empresas }\end{array}$ \\
\hline \multirow[t]{2}{*}{ Chihuahua } & 4 & UACH & Universidad Autónoma de Chihuahua & $\begin{array}{c}\text { Licenciatura en Administración } \\
\text { de Empresas }\end{array}$ \\
\hline & 5 & UACJ & $\begin{array}{l}\text { Universidad Autónoma de Ciudad } \\
\text { Juárez }\end{array}$ & $\begin{array}{l}\text { Licenciatura en Administración } \\
\text { de Empresas }\end{array}$ \\
\hline \multirow{3}{*}{$\begin{array}{l}\text { Distrito } \\
\text { Federal }\end{array}$} & 6 & UNAM & $\begin{array}{c}\text { Universidad Nacional Autónoma de } \\
\text { México } \\
\end{array}$ & Licenciatura en Administración \\
\hline & 7 & IPN & $\begin{array}{c}\text { Instituto Politécnico Nacional, Unidad } \\
\text { Interdisciplinaria de Ingeniería y } \\
\text { Ciencias Sociales y Administrativas } \\
\text { UPICSA }\end{array}$ & $\begin{array}{c}\text { Licenciatura en Administración } \\
\text { Industrial }\end{array}$ \\
\hline & 8 & UVM & $\begin{array}{c}\text { Universidad del Valle de México. } \\
\text { Campus San Rafael, Tlalpán, Lomas } \\
\text { Verdes y Querétaro }\end{array}$ & Licenciatura en Adm \\
\hline Guanajuato & 9 & ITC & Instituto Tecnológico de Celaya & Licenciatura en Administración \\
\hline Hidalgo & 10 & UAEH & $\begin{array}{l}\text { Universidad Autónoma del Estado de } \\
\text { Hidalgo }\end{array}$ & Licenciatura en $\mathrm{Ad}$ \\
\hline Jalisco & 11 & UDG & Universidad de Guadalajara & Licenciatura en Administración \\
\hline México & 12 & UAEM & $\begin{array}{l}\text { Universidad Autónoma del Estado de } \\
\text { México }\end{array}$ & Licenciatura en Administración \\
\hline \multirow[t]{2}{*}{ Nuevo León } & 13 & UANL & Universidad Autónoma de Nuevo León & Licenciatura en Administración \\
\hline & 14 & ITESM & $\begin{array}{c}\text { Instituto Tecnológico y de Estudios } \\
\text { Superiores de Monterrey. Campus Edo. } \\
\text { de México, Monterrey, Guadalajara y } \\
\text { Chiapas }\end{array}$ & $\begin{array}{c}\text { Licenciatura en Administración } \\
\text { de Empresas }\end{array}$ \\
\hline Oaxaca & 15 & ITM & Universidad Tecnológica de la Mixteca & $\begin{array}{c}\text { Licenciatura en Ciencias } \\
\text { Empresariales }\end{array}$ \\
\hline \multirow[t]{2}{*}{ Puebla } & 16 & BUAP & $\begin{array}{c}\text { Benemérita Universidad Autónoma de } \\
\text { Puebla }\end{array}$ & $\begin{array}{c}\text { Licenciatura en Administración } \\
\text { de Empresas }\end{array}$ \\
\hline & 17 & UPAP & $\begin{array}{l}\text { Universidad Popular Autónoma del } \\
\text { Estado de Puebla }\end{array}$ & $\begin{array}{l}\text { Licenciatura en Administración } \\
\text { de Empresas }\end{array}$ \\
\hline $\begin{array}{c}\text { San Luis } \\
\text { Potosí }\end{array}$ & 18 & UASLP & $\begin{array}{c}\text { Universidad Autónoma de San Luis } \\
\text { Potosí }\end{array}$ & Licenciatura en Administración \\
\hline Sinaloa & 19 & ODO & $\begin{array}{l}\text { Universidad de Occidente. Sedes Los } \\
\text { Mochis, Culiacán y Mazatlán }\end{array}$ & $\begin{array}{c}\text { Licenciatura en Administración } \\
\text { de Empresas }\end{array}$ \\
\hline Tamaulipas & 20 & UAT & Universidad Autónoma de Tamaulipas & Licenciatura en Administración \\
\hline \multirow[t]{2}{*}{ Veracruz } & 21 & $\mathbf{U V}$ & $\begin{array}{c}\text { Universidad Veracruzana, Zonas } \\
\text { Veracruz, Orizaba-Córdoba y Xalapa }\end{array}$ & $\begin{array}{l}\text { Licenciatura en Administración } \\
\text { de Empresas }\end{array}$ \\
\hline & 22 & UNIVO & Universidad del Valle de Orizaba & Licenciatura en Administración \\
\hline
\end{tabular}

Fuente: CACECA Consultado en junio de 2005 en: http://www.caceca.org/ 
Anexo 2

Materias básicas

\begin{tabular}{|c|c|c|c|c|c|c|c|}
\hline 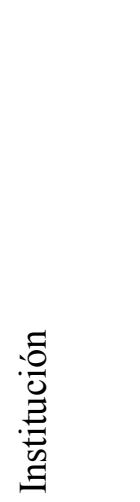 & 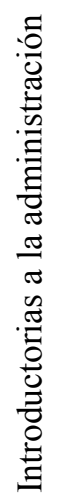 & 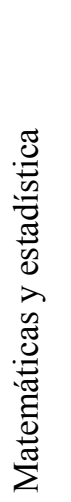 & 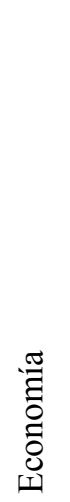 & 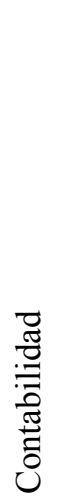 & 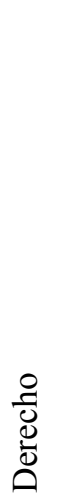 & 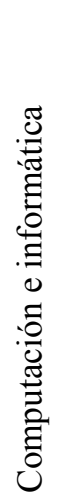 & 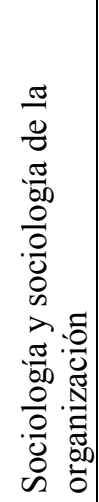 \\
\hline UAA & $\mathrm{X}$ & $\mathrm{X}$ & $X$ & $\mathrm{X}$ & $\mathrm{X}$ & $\mathrm{X}$ & $X$ \\
\hline UABC & $\mathrm{X}$ & $X$ & $X$ & $\mathrm{X}$ & $\mathrm{X}$ & $\mathrm{X}$ & \\
\hline CETYS & $\mathrm{X}$ & $X$ & $X$ & $\mathrm{X}$ & $\mathrm{X}$ & $\mathrm{X}$ & $\mathrm{X}$ \\
\hline UACH & $\mathrm{X}$ & $\mathrm{X}$ & $X$ & $\mathrm{X}$ & $\mathrm{X}$ & $\mathrm{X}$ & $\mathrm{X}$ \\
\hline UACJ & $\mathrm{X}$ & $\mathrm{X}$ & $\mathrm{X}$ & $\mathrm{X}$ & $\mathrm{X}$ & & \\
\hline UNAM & $\mathrm{X}$ & $\mathrm{X}$ & $X$ & $\mathrm{X}$ & $\mathrm{X}$ & $\mathrm{X}$ & $\mathrm{X}$ \\
\hline IPN & $\mathrm{X}$ & $\mathrm{X}$ & $X$ & $\mathrm{X}$ & $\mathrm{X}$ & $\mathrm{X}$ & \\
\hline UVM & $\mathrm{X}$ & $X$ & $X$ & $\mathrm{X}$ & $\mathrm{X}$ & $\mathrm{X}$ & $\mathrm{X}$ \\
\hline ITC & $\mathrm{X}$ & $\mathrm{X}$ & $\mathrm{X}$ & $\mathrm{X}$ & $\mathrm{X}$ & $\mathrm{X}$ & $\mathrm{X}$ \\
\hline UAEH & $\mathrm{X}$ & $X$ & $X$ & $\mathrm{X}$ & $\mathrm{X}$ & $\mathrm{X}$ & $\mathrm{X}$ \\
\hline UDG & $\mathrm{X}$ & $\mathrm{X}$ & $\mathrm{X}$ & $\mathrm{X}$ & $\mathrm{X}$ & $\mathrm{X}$ & \\
\hline UAEM & $\mathrm{X}$ & $X$ & $X$ & $\mathrm{X}$ & $\mathrm{X}$ & $\mathrm{X}$ & \\
\hline UANL & $\mathrm{X}$ & $X$ & $X$ & $\mathrm{X}$ & $\mathrm{X}$ & $\mathrm{X}$ & $\mathrm{X}$ \\
\hline ITESM & $X$ & $X$ & $X$ & $\mathrm{X}$ & $\mathrm{X}$ & $\mathrm{X}$ & \\
\hline UTM & $\mathrm{X}$ & $\mathrm{X}$ & $X$ & $\mathrm{X}$ & $\mathrm{X}$ & $\mathrm{X}$ & \\
\hline BUAP & $\mathrm{X}$ & $X$ & $X$ & $\mathrm{X}$ & $\mathrm{X}$ & $\mathrm{X}$ & $X$ \\
\hline UPAP & $X$ & $\mathrm{X}$ & $X$ & $X$ & $\mathrm{X}$ & $\mathrm{X}$ & $X$ \\
\hline UASLP & $\mathrm{X}$ & $\mathrm{X}$ & $X$ & $\mathrm{X}$ & $\mathrm{X}$ & $\mathrm{X}$ & $\mathrm{X}$ \\
\hline UDO & $\mathrm{X}$ & $\mathrm{X}$ & $\mathrm{X}$ & $\mathrm{X}$ & $\mathrm{X}$ & $\mathrm{X}$ & \\
\hline UAT & $\mathrm{X}$ & $\mathrm{X}$ & $\mathrm{X}$ & $\mathrm{X}$ & $\mathrm{X}$ & $\mathrm{X}$ & \\
\hline UV & $\mathrm{X}$ & $\mathrm{X}$ & $X$ & $\mathrm{X}$ & $\mathrm{X}$ & $\mathrm{X}$ & \\
\hline \multirow[t]{2}{*}{ UNIVO } & $X$ & $\mathrm{X}$ & $X$ & $\mathrm{X}$ & $\mathrm{X}$ & $\mathrm{X}$ & $\mathrm{X}$ \\
\hline & 22 & 22 & 22 & 22 & 22 & 21 & 12 \\
\hline
\end{tabular}


La formación de administradores

en el nuevo entorno internacional

Anexo 3

Proceso administrativo y áreas funcionales

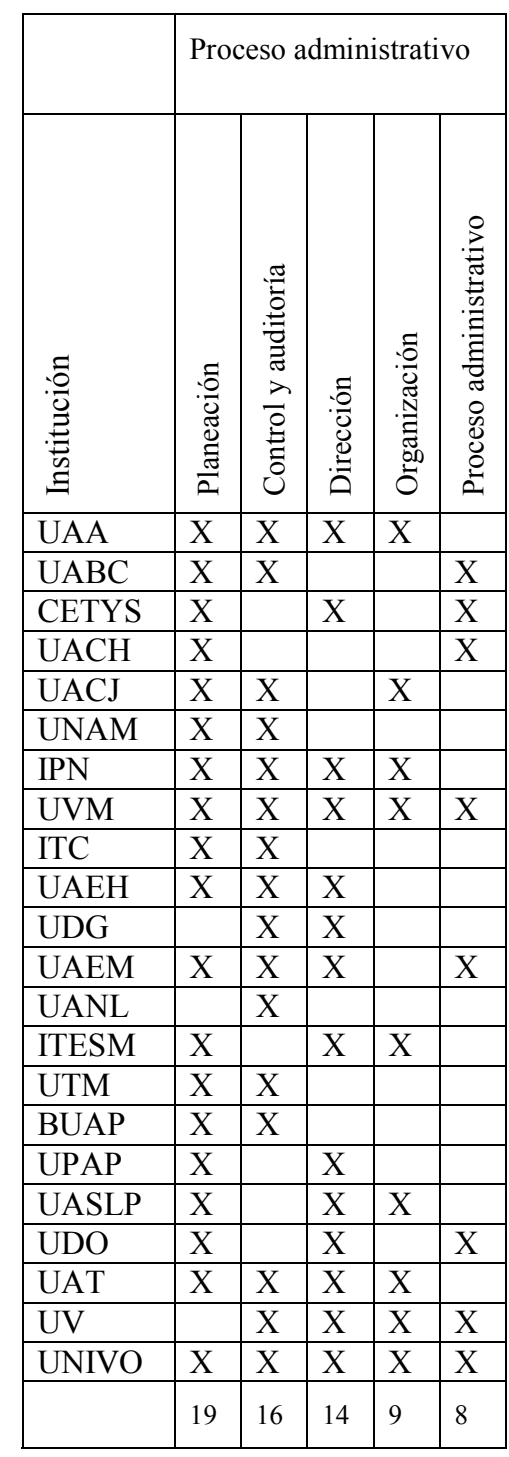

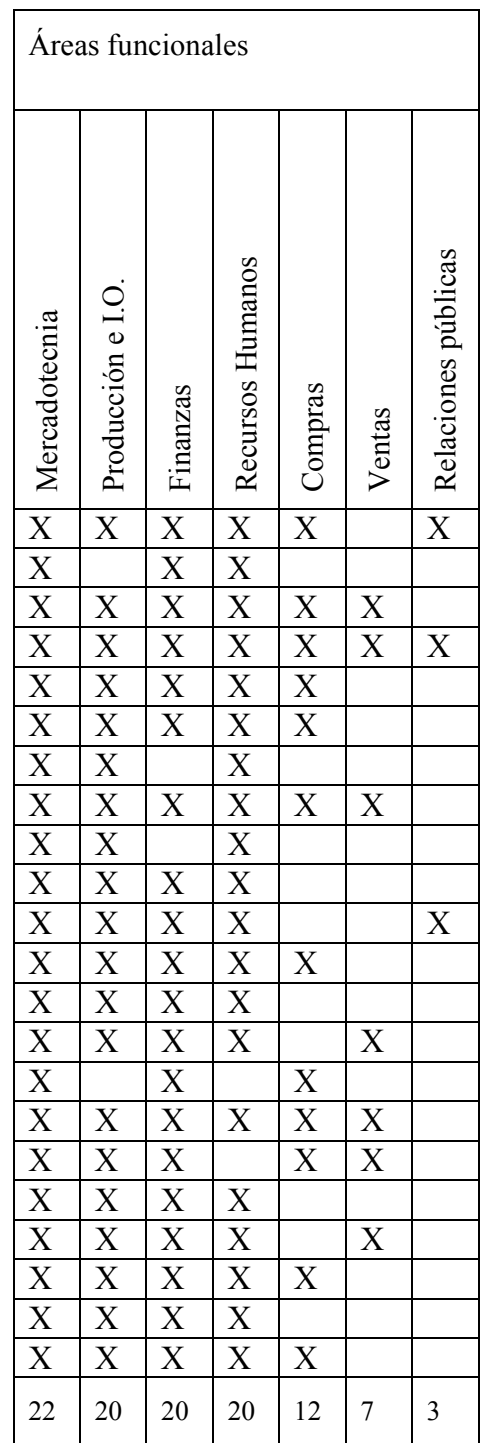

No. 220, septiembre-diciembre 2006 
José Luis Pariente Fragoso

\section{Anexo 4}

Otras materias

\begin{tabular}{|c|c|c|c|c|c|c|c|c|c|c|c|c|c|c|c|c|c|c|c|c|c|}
\hline 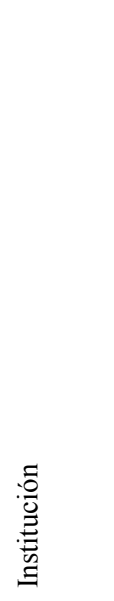 & 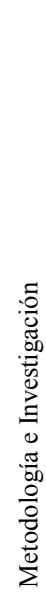 & 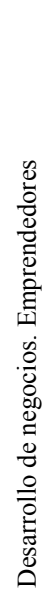 & . & 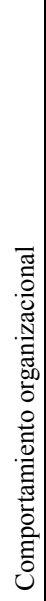 & 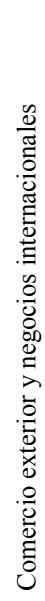 & 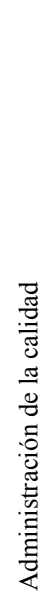 & $\sum_{i}^{\infty}$ & $\left|\begin{array}{c|}\mathscr{v} \\
\frac{\mathscr{d}}{b 0} \\
\Xi\end{array}\right|$ & 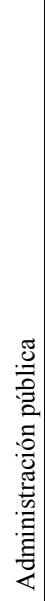 & 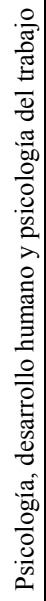 & 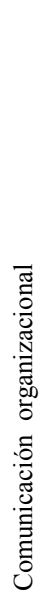 & 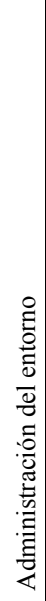 & 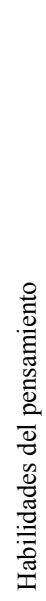 & 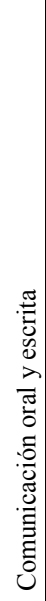 & 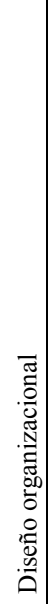 & $\begin{array}{l}0 \\
\stackrel{0}{w} \\
0 \\
\Xi \\
\Xi \\
\Xi\end{array}$ & 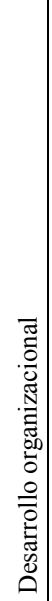 & 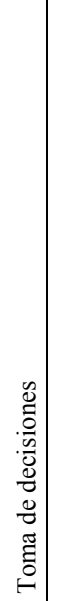 & 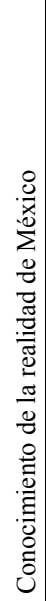 & 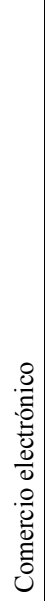 & 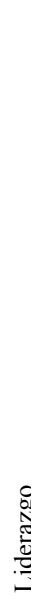 \\
\hline UAA & $X$ & $\mathrm{X}$ & $\mathrm{X}$ & & $X$ & & $X$ & & $\mathrm{X}$ & $\mathrm{X}$ & & & & & & $\mathrm{X}$ & & $X$ & & & \\
\hline $\mathrm{UABC}$ & $\mathrm{X}$ & & $\mathrm{X}$ & & & & $\mathrm{X}$ & & $\mathrm{X}$ & & & $\mathrm{X}$ & $\mathrm{X}$ & & $\mathrm{X}$ & $\mathrm{X}$ & $\mathrm{X}$ & & & & $\mathrm{X}$ \\
\hline CETYS & $\mathrm{X}$ & $\mathrm{X}$ & $X$ & $\mathrm{X}$ & $\mathrm{X}$ & & $X$ & & $\mathrm{X}$ & & $\mathrm{X}$ & $\mathrm{X}$ & $X$ & $\mathrm{X}$ & & & & & & & \\
\hline UACH & & $X$ & & $\mathrm{X}$ & & & & & & & $\mathrm{X}$ & & $X$ & & & & & & & $\mathrm{X}$ & \\
\hline UACJ & & & & $\mathrm{X}$ & & $X$ & & & & & & & & & & & $\mathrm{X}$ & & & & \\
\hline UNAM & $X$ & & $\mathrm{X}$ & & & & $\mathrm{X}$ & & $\mathrm{X}$ & $\mathrm{X}$ & & & & & & & & & & & \\
\hline IPN & & $\mathrm{X}$ & $\mathrm{X}$ & $\mathrm{X}$ & & $\mathrm{X}$ & & & & & $X$ & & & & & & & & & & \\
\hline UVM & $\mathrm{X}$ & $X$ & $X$ & & & $\mathrm{X}$ & & & & & $X$ & & $\mathrm{X}$ & & & & & & $\mathrm{X}$ & & \\
\hline ITC & $\mathrm{X}$ & $X$ & $X$ & $\mathrm{X}$ & & $\mathrm{X}$ & & & & & $\mathrm{X}$ & $\mathrm{X}$ & & & $\mathrm{X}$ & & & & & & \\
\hline UAEH & $\mathrm{X}$ & $\mathrm{X}$ & $X$ & & & $\mathrm{X}$ & & $\mathrm{X}$ & & $\mathrm{X}$ & & & & & $\mathrm{X}$ & $\mathrm{X}$ & & & & & $\mathrm{X}$ \\
\hline UDG & $X$ & $\mathrm{X}$ & $\mathrm{X}$ & $\mathrm{X}$ & $\mathrm{X}$ & $X$ & & & $\mathrm{X}$ & & & & & & $X$ & & $\mathrm{X}$ & & & & \\
\hline UAEM & $\mathrm{X}$ & & & $\mathrm{X}$ & $\mathrm{X}$ & & & & $\mathrm{X}$ & & $\mathrm{X}$ & & & & & & & & $\mathrm{X}$ & $\mathrm{X}$ & \\
\hline UANL & $\mathrm{X}$ & $\mathrm{X}$ & $\mathrm{X}$ & & & & & & & & & $\mathrm{X}$ & & $\mathrm{X}$ & & & & & & & \\
\hline ITESM & & $X$ & & & $\mathrm{X}$ & $X$ & & $X$ & $\mathrm{X}$ & $\mathrm{X}$ & & & & & & & & $\mathrm{X}$ & & $\mathrm{X}$ & $\mathrm{X}$ \\
\hline UTM & $\mathrm{X}$ & & & & & & & $\mathrm{X}$ & & $\mathrm{X}$ & & & & & & & $\mathrm{X}$ & $X$ & & & \\
\hline BUAP & $\mathrm{X}$ & $\mathrm{X}$ & $\mathrm{X}$ & $\mathrm{X}$ & & $\mathrm{X}$ & $X$ & $X$ & & & & & & $\mathrm{X}$ & & $\mathrm{X}$ & & & & & \\
\hline UPAP & $\mathrm{X}$ & & $\mathrm{X}$ & $\mathrm{X}$ & $\mathrm{X}$ & & & $\mathrm{X}$ & & & & $\mathrm{X}$ & & & & & $\mathrm{X}$ & & & & \\
\hline UASLP & & $\mathrm{X}$ & & & $\mathrm{X}$ & & & & & & & & $\mathrm{X}$ & $\mathrm{X}$ & & & & & & & \\
\hline UDO & $X$ & & & $\mathrm{X}$ & $\mathrm{X}$ & & & $X$ & & & & $\mathrm{X}$ & & $\mathrm{X}$ & $\mathrm{X}$ & & & $\mathrm{X}$ & & & \\
\hline UAT & & $\mathrm{X}$ & & & & & $\mathrm{X}$ & $\mathrm{X}$ & & $\mathrm{X}$ & $X$ & $\mathrm{X}$ & $\mathrm{X}$ & & & & & & $\mathrm{X}$ & & \\
\hline UV & $X$ & $\mathrm{X}$ & & $\mathrm{X}$ & $X$ & & $\mathrm{X}$ & $\mathrm{X}$ & & & & & $\mathrm{X}$ & $\mathrm{X}$ & & $\mathrm{X}$ & & & & & \\
\hline \multirow[t]{2}{*}{ UNIVO } & $X$ & & & $\mathrm{X}$ & & & $X$ & & $\mathrm{X}$ & $\mathrm{X}$ & & & & & $\mathrm{X}$ & & & & & & \\
\hline & 16 & 14 & 12 & 12 & 9 & 8 & 8 & 8 & 8 & 7 & 7 & 7 & 7 & 6 & 6 & 5 & 5 & 4 & 3 & 3 & 3 \\
\hline
\end{tabular}

